\title{
İ acreditacion de las carreras de la FACEE en el escenario de la universidad peruana
}

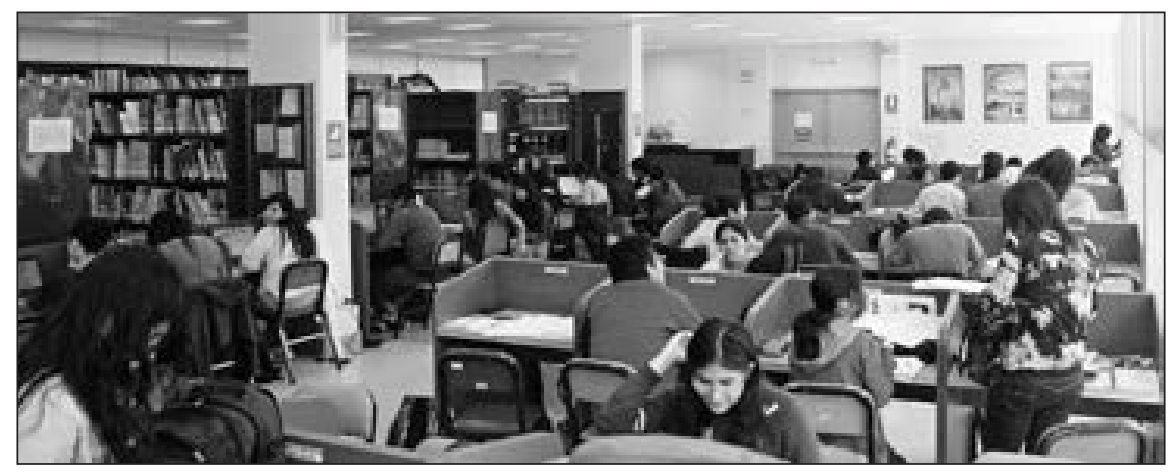

Por: Jesús Hidalgo Ortega

El presente artículo expone la importancia del proceso de acreditación para elevar la calidad de la Educación Universitaria. Asimismo, destaca el importante papel que desempeñan el Sistema Nacional de Evaluación, Acreditación y Certificación de la Calidad Educativa (Sineace) y el Consejo Nacional de Evaluación, Acreditación y Certificación de la Calidad de la Educación Universitaria (CONEAU) en este proceso. Igualmente se expone la destacada labor que desarrollan los Comités Internos de Acreditación de las cinco carreras académico profesionales de Facultad de Ciencias Económicas y Empresariales.

Palabras claves: acreditación, calidad, educación, docentes, autoevaluación, carreras.

\section{Introducción}

Nuestro país se hallaba muy rezagado en el tema de la acreditación de la calidad de la Educación, en relación a los esfuerzos internacionales y aun de la región. Tal es así que recién en 2006 se dio la Ley del Sistema Nacional de Evaluación, Acreditación y Certificación de la Calidad de la Educación, SINEACE, y solo en 2007 se crea por la Ley respectiva el órgano ejecutor para la educación universitaria, el CONEAU, Consejo Nacional de Evaluación, Acreditación y Certificación de la Calidad de la Educación Universitaria. Se estableció allí el modelo de calidad general, aprobándose los estándares correspondientes a educación únicamente en 2011, cuatro años después. La norma legal indicó la obligatoriedad de la acreditación de las carreras de Salud, Educación y Derecho, estableciendo los estándares de calidad para dichas carreras. Los estándares para las carreras correspondientes a la FACEE, administración (para Administración y Gerencia, Administración de Negocios Globales, y Turismo, Hotelería y Gastronomía), Contabilidad y Economía se establecieron tardíamente en versión definitiva en 2012 y 2013. Por ejemplo, los estándares para la acreditación de la Carrera Profesional de Contabilidad se aprobaron recién en 2012, siendo publicada el 1 de junio de 2012 en el diario oficial "El Peruano".

La Universidad Ricardo Palma, desde el 2002, dio los pasos para adoptar un Modelo de Autoevaluación, siendo una de las universidades pioneras en el Perú. En efecto, desde ese año la Universidad hizo grandes esfuerzos para establecer un modelo propio de autoevaluación para la acreditación de la calidad de sus carreras profesionales; sin embargo, al no existir referentes nacionales, salvo los planteados en otras universidades públicas y privadas, sin el consenso general necesario, el modelo de autoevaluación de la URP se dirigió no a la acreditación, al no existir la entidad acreditadora pública nacional, sino a la mejora continua. Estos esfuerzos representaron una verdadera sensibilización y ejercicio previo, pero su carácter particular y voluntario en medio de la incertidumbre restaba fuerza a sus recomendaciones y obligaciones. En este contexto, las Facultades de Medicina Humana e Ingeniería recurrieron a la acreditación internacional de sus carreras profesionales, significando un paso muy importante para la universidad. 


\section{Inicio y organización del proceso de acreditación}

Nuestra Facultad inicia el Proceso de Acreditación de sus Carreras cuando el Consejo de Facultad implementó los Comités Internos de Autoevaluación de las 5 carreras profesionales a acreditarse, y un Comité de Acreditación central, lo cual fue aprobado por el Consejo Universitario el 28 de febrero de 2012, mediante acuerdo de Consejo Universitario $\mathrm{N}^{\circ} 0471$ - 2012. Después de un proceso previo de organización, en el cual se incluyeron nuevos miembros a los Comités, este acuerdo de la Universidad Ricardo Palma fue informado al SINEACE, mediante el Oficio No 0846-2012- URP-R, recepcionado el 24 de Octubre de 2012, por el cual comunicaba a dicho organismo el inicio de las actividades para el proceso de Acreditación; y se da a conocer la composición de los Comités Técnicos Internos por Carrera Profesional y se solicita la capacitación sobre la metodología de autoevaluación según el modelo de CONEAU, de las Carreras Profesionales de la Facultad de Ciencias Económicas y Empresariales:

- Administración y Gerencia

- Administración de Negocios Globales

- Contabilidad y Finanzas

- Economía

- Turismo, Hotelería y Gastronomía.

El proceso se inició y planificó como se explica en el contenido siguiente, pero la fase final de la autoevaluación se fija el 16 de enero de 2014, cuando el señor Rector de la Universidad Ricardo Palma, doctor Iván Rodríguez Chávez, enfatiza ante la comunidad, la necesidad de la acreditación nacional de las carreras profesionales de la Universidad, disponiendo que las Facultades culminen sus procesos de autoevaluación en plazo perentorio, conforme al modelo de acreditación nacional del SINEACE.

\section{Planificación del proceso de autoevaluación}

El Proceso de acreditación de la Facultad de Ciencias Económicas y Empresariales es presidido por el señor decano de la Facultad, Dr. Jesús Hidalgo Ortega, como lo establece la propia normativa del CONEAU - SINEACE. Él fue designado por el Consejo de Facultad y ratificado por el Consejo Universitario; y con el apoyo del Comité Técnico de Autoevaluación de la FACEE, se planificó la coordinación con los Comités Internos de Acreditación de las cinco escuelas académico profesionales, las metas e indicadores, y los plazos de los avances conforme a los Proyectos de Acreditación presentados por cada Comité Interno de la Escuelas.

Funcionalmente, conforme al ordenamiento de la Universidad, el Comité Interno de Acreditación de cada Escuela dependía del Comité Interno de la Facultad, y este a su vez del Consejo de Facultad y consejo universitario, debido a que la Acreditación ameritaba la instauración de documentos normativos en la Facultad, conforme al Modelo de Calidad de CONEAU. Gran parte de los documentos pasaron por el proceso de aprobación a través de los órganos de gobierno, en especial del Consejo de Facultad y el Consejo Universitario.

Asimismo, el Flujo procesual documentario de la Acreditación se organizó de la siguiente forma, como se aprecia en el gráfico $\mathrm{N}^{\circ} 1$.

\section{Gráfico $\mathrm{N}^{\circ}$ 1: Flujograma documental de Acreditación}

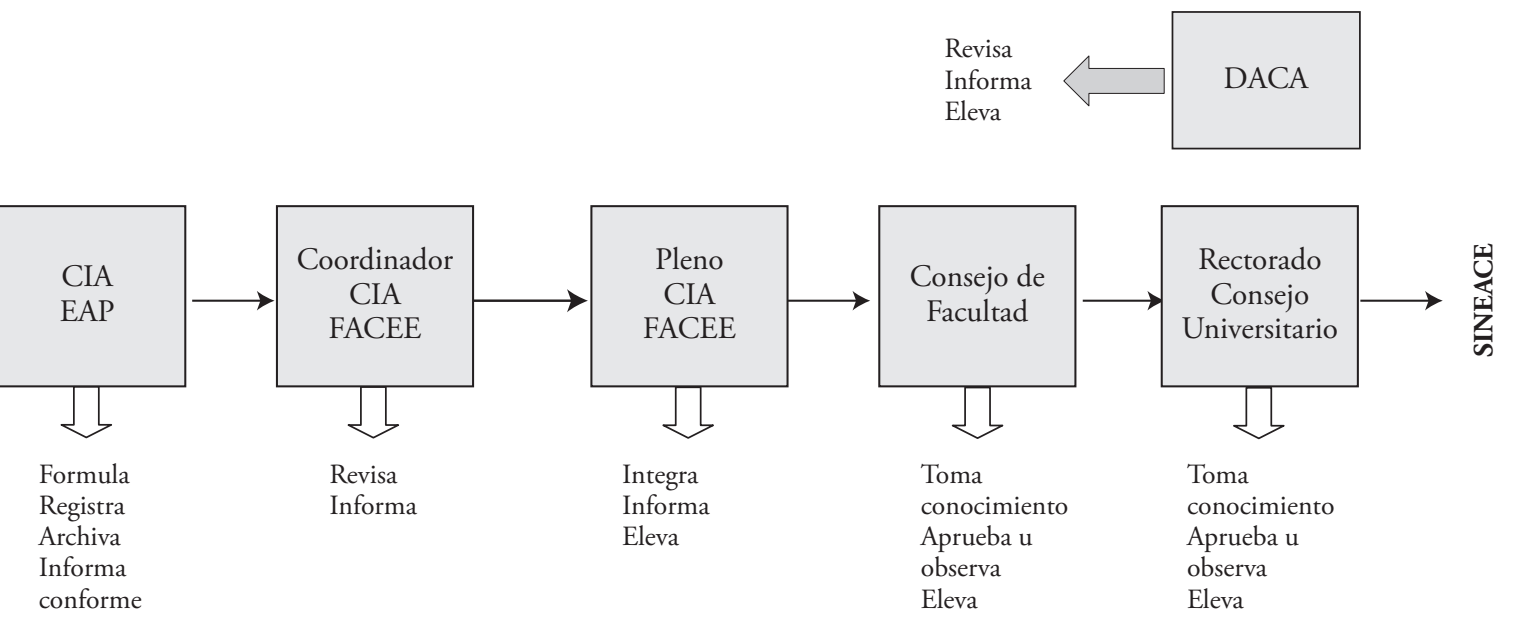

CIA EAP : Comité Interno de autoevaluación por Escuela (carrera)

CIA FACEE : Comité Interno de Autoevaluación central (FACEE)

DACA : O.Desarrollo Académico, Calidad y Acreditación 
En la normativa de la acreditación nacional son los Comités Internos de Autoevaluación de las carreras profesionales, de las Escuelas, los responsables directos en la conducción del proceso de autoevaluación; sin embargo, en el caso de la FACEE se hacía necesario, en una fase previa, una instancia intermedia que en este caso fue el Comité Interno de Autoevaluación de la FACEE, presidido por el señor decano de la Facultad. Esto se justifica porque en nuestra Facultad muchas funciones están concentradas en oficinas facultativas, por ejemplo, Grados y Títulos, Registros Académicos y Matrícula, Biblioteca, Laboratorio de Informática, Instituto de Investigación, Extensión Universitaria, entre otros. Además, las Escuelas están en pleno proceso de desarrollo organizacional, por lo cual muchas funciones la ejerce la Facultad.

El Comité Interno de Autoevaluación de la FACEE, cumplió con énfasis en el segundo semestre del 2012 y durante el 2013 las labores de difusión de las normas del proceso de acreditación nacional e internacional, proponiendo cronogramas y procedimientos, requiriendo información y formulando diagnósticos previos sobre la calidad de las actividades de la Facultad. El comité coordinó permanentemente el proceso con la Oficina de Desarrollo Académico, Calidad y Acreditación.

La realidad demostró la necesidad de este "Comité Central", pues en adición, existieron demoras por los obstáculos ya expuestos en constituir los comités internos de las carreras, comités que se reestructuraron en más de una ocasión en el caso de algunas escuelas, a tal punto que recién a inicios del año 2014 determinadas escuelas habían nombrado su comité interno definitivo. Además, la limitación de los recursos restringió y demoró la asignación de horas no lectivas, retrasando las labores. Por lo demás, las Escuelas debieron reforzar sus Comités con docentes recién ingresados a la Universidad, quienes tomaron la posta, debiendo dedicar tiempo a tomar conocimiento del estado del proceso, supliendo con mucho esfuerzo la falta de experiencia.

Así, el Comité Interno de la FACEE fue vital en el proceso de iniciación de la autoevaluación, sirviendo como "catalizador" del proceso, tratando en todo momento de moderar y conducir las tendencias autocentristas de las Escuelas, llevándola siempre a la cooperación y al logro de las sinergias. Posteriormente, como era lógico, fueron los Comités Internos de las Escuelas las que tomaron la mayor actividad, como responsables directos de la autoevaluación.
Una vez tomado este direccionamiento, la Facultad debió organizar sus cuadros en forma acelerada, en medio de restricciones presupuestarias que en un principio no permitieron una organización pronta del proceso de autoevaluación. Algunos miembros de la Facultad mostraron inicialmente su incredulidad, debido a los grandes obstáculos, restricciones de recursos y tiempos y cambios en el estado de cosas que requería el proceso de acreditación; sin embargo, ellos mismos llegaron a ser posteriormente grandes artífices del proceso. Muchos tardaron en comprender que la autoevaluación no era un asunto de "expertos", sino un ejercicio participativo de reflexión sobre las actividades de la comunidad universitaria en cada Escuela y en toda la Facultad, que involucraba a todos. La Universidad, a su vez, estaba en pleno proceso de actualización de sus instrumentos de gestión, como el Reglamento General y el Plan Estratégico, y nuestros planes curriculares no se habían revisado conforme a lo planificado, datando la última actualización de seis años atrás.

\section{Los retos de la acreditación de la FACEE}

El reto de acreditar cinco carreras profesionales al mismo tiempo no tenía antecedentes; existían además, obstáculos a la acreditación, entre los cuales citamos:

- Progresiva comprensión de la autoevaluación como un proceso interno, propio de cada estamento de la FACEE y las Escuelas. Es decir, fue un proceso arduo comprender que la planificación de las actividades, la formulación de metas específicas, y la evaluación de las actividades, son funciones propia de cada área organizacional, con autonomía, y no una imposición "externa” de la acreditación.

- Esto se unió a una visión parcial de la acreditación como "fin en sí misma", o como proceso "técnico" sin relación con la mejora contínua de las carreras profesionales de la Facultad.

- La cultura de planificación, autoevaluación y aseguramiento de la calidad estaba en sus inicios.

- Un proceso de planificación estratégica a nivel universidad en desarrollo, la cultura de autoevaluación y el proceso de acreditación exigen imperativamente optimizar este proceso

- Falta de cuadros preparados en autoevaluación y acreditación. 
- Recursos limitados

El reto era imposible de superar con un enfoque individual, carrera por carrera, una por una; era necesario y vital conseguir una sinergia de esfuerzos entre las cinco escuelas profesionales y el decanato. Este, como cabeza de la Facultad, se dedicó a lograrla, elevando los esfuerzos de los cinco comités internos de autoevaluación, por encima de los intereses personales y de grupo, las diferencias personales y de carácter, la soberbia y otras actitudes que debieron deponerse en aras del bien común. En especial, a beneficio de los estudiantes de toda la Facultad.

La estrategia de la sinergia se dio en tres formas:

1. Reuniones y exposiciones conjuntas de los comités de autoevaluación de las EAPs, para compartir avances, experiencias y dudas.

2. Intercambio de los avances y documentos entre los Comités de las escuelas.

3. Preparación por parte de los docentes convocados por el Decanato de los documentos comunes a las EAP, como el Plan Estratégico, el Manual y Reglamento de Organización y Funciones, y los documentos sistémicos, como por ejemplo, y entre muchos otros, el Sistema de evaluación de la Enseñanza - Aprendizaje y el Sistema de Gestión de la Calidad.

\section{La integración de los grupos de interés}

Como una demanda del modelo de acreditación del CONEAU, las Escuelas Académico - Profesionales de la FACEE conformaron los Comités Consultivos de los Grupos de Interés, que constituyen la incorporación dentro de la gestión de la carrera de todos los sectores sociales que tienen una relación e interés con nuestras carreras, como son las empresas, los gremios empresariales, las instituciones públicas, los colegios profesionales, los sectores organizados de la sociedad civil, etc. Entre ellos se incluyen asimismo a los egresados. La conformación de los Comités Consultivos permite reforzar los vínculos de la Carrera Profesional con la sociedad, fortaleciendo la pertinencia de la formación profesional, investigación, extensión universitaria y proyección social. En este sentido, permite facilitar las consultas para la revisión permanente de los perfiles de egresados y para el adecuado planeamiento curricular, entre muchos otros aspectos. Los comités consultivos participan asimismo en el direccionamiento estratégico de las carreras.

Las Escuelas iniciaron el proceso de selección de los integrantes de los comités consultivos, proceso que permitió la convocatoria de representantes de organizaciones de gran proyección en la sociedad, de personalidades de gran influencia del mundo empresarial, gubernamental, social y académico. La conformación de los Comités fue aprobada por el Consejo de Facultad y por el Consejo Universitario.

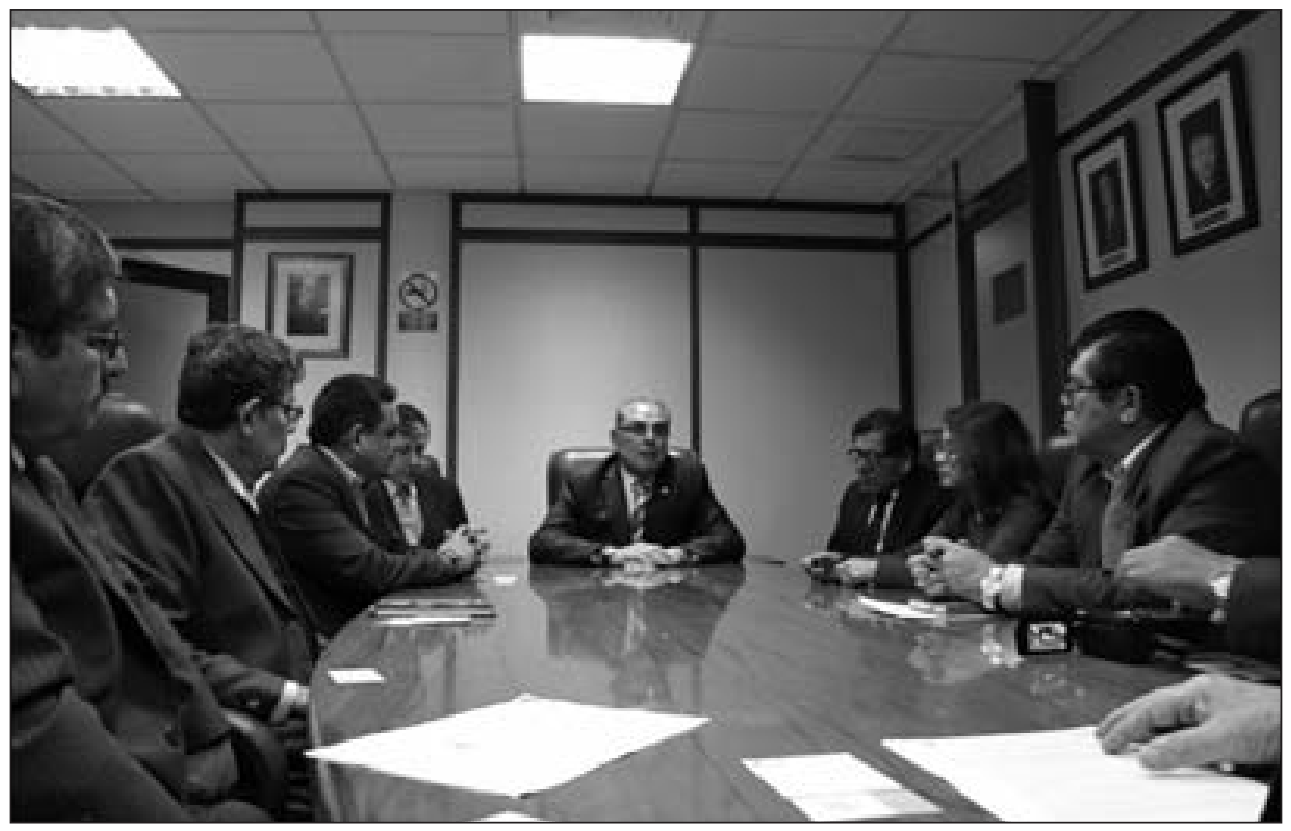

El Comité Consultivo de la EAP de Administración y Gerencia, en la sesión de instalación de la misma, en la Sala de Sesiones del Consejo de Facultad (27- 08- 2014), con la presencia del seńor director de la EAP Carlos Jungbluth Voysest y el señor decano de la FACEE Jesús Hidalgo Ortega. 
La Facultad de Ciencias Económicas de la Universidad Ricardo Palma debe expresar su más alto reconocimiento a las personalidades del mundo empresarial, político, científico, profesional y social que están participando en los Comités Consultivos de sus Carreras Profesionales.

\section{El sistema de aseguramiento de la calidad de la} FACEE

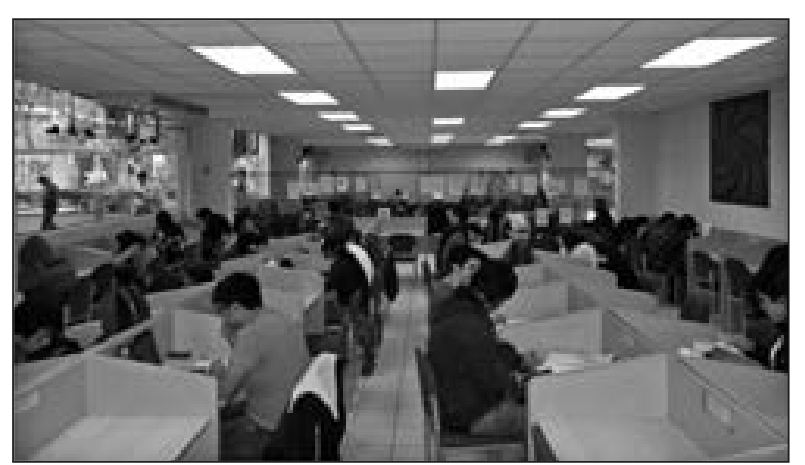

Biblioteca Especializada FACEE. Pabellón "D”

Como resultado de la autoevaluación de las carreras profesionales, la FACEE, en su conjunto, ha podido presentar un diagnóstico completo de los procesos de enseñanza - aprendizaje, investigación, extensión universitaria y proyección social, inquiriendo las perspectivas de los estudiantes, egresados, docentes, servidores administrativos, grupos de interés y directivos. Ello ha permitido identificar nuestras principales fortalezas y deficiencias, nuestras oportunidades y riesgos, lo cual está contenido en los Informes Finales de Autoevaluación de cada carrera; a partir de ello se han propuesto los rumbos de acción consecuentes, reseñados en los Planes de Mejora.

\section{Gráfico $\mathrm{N}^{\circ}$ 2: Principales sistemas del Modelo de Calidad y su relación mutua}

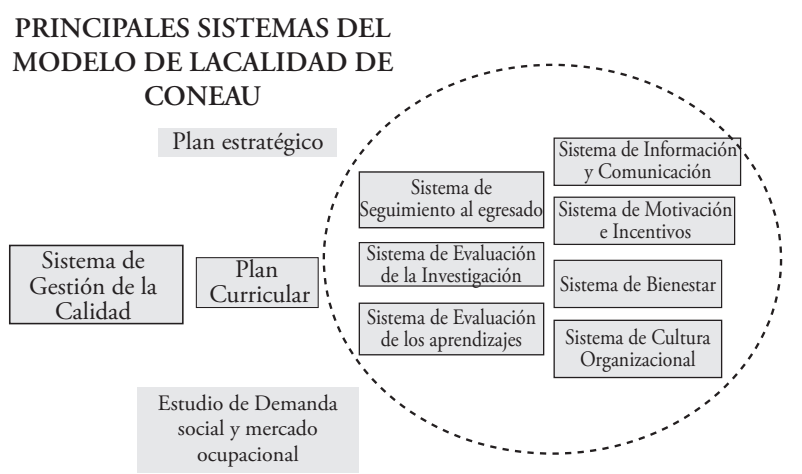

A manera de síntesis, ¿qué duda cabe que a nuestra Facultad le falta investigación a nivel docente y de estudiante? ¿qué duda cabe que muchos estudiantes rehúyen preparar una tesis para obtener el grado y el título profesional? Además, requerimos una investigación ligada a la formación profesional a través del currículo, y con líneas de investigación ya determinadas con antelación. Lo mismo puede decirse de la Extensión Universitaria y Proyección social, que son una fuente inagotable de aprendizajes personales, de vinculación con la comunidad y de responsabilidad social institucional y personal.

A los estudiantes de nuestras carreras, asimismo, les va a ayudar mucho una tutoría sistemática, que sea un acompańamiento efectivo al estudiante, tanto en el aspecto académico como en el personal, para el direccionamiento de sus planes de vida y carrera, disminuyendo así la repitencia y la deserción, que retrasan y en algunos casos truncan la vida profesional del estudiante, y crea además inestabilidad en la institución.

Los egresados deben tener a partir de ahora una mayor relación con la Escuela y con la FACEE, y los señores docentes deben percibir una optimización de su dedicación a fin de reforzar su participación en la investigación, la extensión universitaria y, en general, en la mejora continua de la Facultad.

Las carreras de la FACEE necesitan integrarse con los grupos de interés, como gremios empresariales, empresas, instituciones públicas, organizaciones no gubernamentales, colegios profesionales, que son los usuarios finales en tanto empleadores de nuestros egresados.

Valoración de lo alcanzado en el proceso de autoevaluación

1. Los comités internos de acreditación han culminado ya con la etapa de Autoevaluación, completando la redacción del Informe de Autoevaluación correspondiente, incluyendo los planes de mejora respectivos.

2. La Primera acreditación nacional es totalmente factible, una vez completados los informes de Autoevaluacion de las carreras, siendo el mayor reto el de perseverar en los compromisos y planes de mejora adoptados para el mejor logro de la calidad educativa. 
3. La primera acreditacion es la que señala los sistemas de los procesos de las carreras; su cumplimiento se verificará en el plazo de tres años, en la segunda acreditación o reacreditación. Por lo cual, todos los sistemas han sido aprobados por los órganos de gobierno correspondientes como normas de la Facultad, y se deben tomar todas las medidas para su implementación garantizando los recursos del caso.

4. Una vez que se culmine la acreditación nacional, el paso de la acreditación internacional es el siguiente, y está totalmente facilitado por los contactos ya tomados con las acreditadoras internacionales y por la acreditación nacional en marcha.

\section{Beneficios alcanzados mediante el proceso de autoevaluación}

Los aspectos específicos logrados mediante la autoevaluación son:
- Un mayor conocimiento de la realidad de la Facultad, con un diagnóstico por carreras profesionales que permitan la toma de decisiones oportuna e informada.

- Una mayor conciencia por parte de la comunidad de la Facultad de nuestras fortalezas, debilidades, riesgos y oportunidades, de las ventajas de la planificación y la factibilidad de la mejora continua.

- Direccionamiento estratégico de la Facultad a través del Plan Estratégico de la Facultad formulado por primera vez, lo que permite un planeamiento operativo preciso que va a garantizar la evaluación objetiva de los procesos, la determinación de las deficiencias y la mejora continua.

- Formulación del Manual y Reglamento de Organización y Funciones, que ayudará al proceso de ordenamiento y alineación con los objetivos estratégicos de la Facultad.

\section{Cronología del proceso de autoevaluación y acreditación de las carreras profesionales de la FACEE}

\begin{tabular}{|c|c|}
\hline Febrero, 28, 2012 & $\begin{array}{l}\text { Aprobación por Consejo Universitario de Comités Internos de Autoevaluación FACEE y } \\
05 \text { EAP. }\end{array}$ \\
\hline Junio, 19,2012 & Visita de acreditadora internacional CACECA a la FACEE. \\
\hline Octubre, 19, de 2012 & Instalación de los Comités Internos de Autoevaluación recompuestos. \\
\hline Octubre, 24, de 2012 & $\begin{array}{l}\text { Comunicación a CONEAU de la apertura del proceso de autoevaluación para la } \\
\text { acreditación nacional de } 5 \text { carreras de la FACEE }\end{array}$ \\
\hline Marzo - julio 2013 & Diagnóstico previo a la autoevaluación de las 5 carreras profesionales. \\
\hline Agosto, 06 de 2013 & Capacitación de CONEAU a los Comités Internos de las EAP de la FACEE. \\
\hline Octubre, 10 de 2013 & $\begin{array}{l}\text { Consejo Universitario aprueba el proyecto de acreditación internacional de la FACEE con } \\
\text { CACECA, CONAET y CONACE. }\end{array}$ \\
\hline Enero, 16 de 2014 & $\begin{array}{l}\text { El señor rector solicita a todas las Facultades de la URP culminar su autoevaluación en } \\
\text { plazo próximo perentorio. }\end{array}$ \\
\hline Febrero de 2014 & Aprobación de los Proyectos de Acreditación nacional de las EAP de la FACEE. \\
\hline Marzo de 2014 & Inicio del proceso de sensibilización por carrera profesional. \\
\hline Marzo 19, 2014 & $\begin{array}{l}\text { Consejo de Facultad de la FACEE designa a los señores docentes encargados de la } \\
\text { formulación de normas sistémicas comunes a la FACEE }\end{array}$ \\
\hline Abril, 22, de 2014 & $\begin{array}{l}\text { Consejo Universitario de la Universidad Ricardo Palma aprueba los Comités Consultivos } \\
\text { de las Carreras Profesionales de la FACEE }\end{array}$ \\
\hline Marzo a julio de 2014 & Consejo de Facultad FACEE aprueba las normas sistémicas. \\
\hline Junio - julio de 2914 & $\begin{array}{l}\text { Aplicación de las encuestas para la autoevaluación a docentes, estudiantes, personal } \\
\text { administrativo y egresados. }\end{array}$ \\
\hline Julio, 21 de 2014 & $\begin{array}{l}\text { Presentación preliminar de los Informes Finales de Autoevaluación de las carreras de la } \\
\text { FACEE al señor Rector. }\end{array}$ \\
\hline Agosto 28 y 29 de 2014 & $\begin{array}{l}\text { Visita Técnica de la Oficina de Desarrollo Académico, Calidad y Acreditación, con presencia } \\
\text { del señor Rector, a las EAP de Administración y Gerencia y Contabilidad y Finanzas }\end{array}$ \\
\hline Octubre de 2014 & Presentación de los Informes Finales de Autoevaluación de 3 Carreras Profesionales FACEE \\
\hline
\end{tabular}


- Ordenamiento de los procesos de Investigación, Proyección Social y Extensión Universitaria, que deben formularse a través de proyectos, con objetivos y metas explícitos, que permitan una mayor retroalimentación y mejora. Esto incluye la normalización e internacionalización de la revista de investigación de la Facultad "Calidad y Creatividad". También se aporta el Reglamento de Propiedad Intelectual de la Facultad, tendiente a alentar y proteger la creación científica de nuestros docentes y estudiantes.

- Establecimiento del Gabinete pedagógico, sistema que debe dirigir y coordinar los procesos de enseñanza aprendizaje en sus fases de planeación, ejecución y evaluación, entre otros muchos aspectos, con herramientas tales como el Sistema de Evaluación del Aprendizaje.

- Establecimiento de la tutoría en forma sistemática, en beneficio del estudiante y su plan de vida y carrera,

- Establecimiento de los comités consultivos de las Carreras Profesionales.

- Normas precisas para la planificación curricular, provista por los estándares del modelo de calidad de
SINEACE que refuerzan la pertinencia del proceso curricular.

- Formulación del Estudio de demanda social y mercado ocupacional para 6 carreras profesionales de la Facultad, que permite un mejor direccionamiento a nuestra reformulación curricular y a las actividades promocionales de nuestras carreras.

\section{Conclusiones y comentarios}

1. La planificación estratégica de la Universidad y la acreditación deben ir de la mano. Ambos procesos se integrarán mediante la inclusión de los planes de mejora y las recomendaciones de la Acreditación en la planificación estratégica y, también, en la operativa.

2. El modelo de aseguramiento de la calidad propuesto tiene un carácter sistémico y holístico, pues todas las 3 dimensiones y los 9 factores planificación de la carrera, enseñanza - aprendizaje, investigación, extensión universitaria y proyección social, docentes, infraestructura y equipamiento, bienestar, recursos financieros, y grupos de interés-, se vinculan, relacionan y condicionan

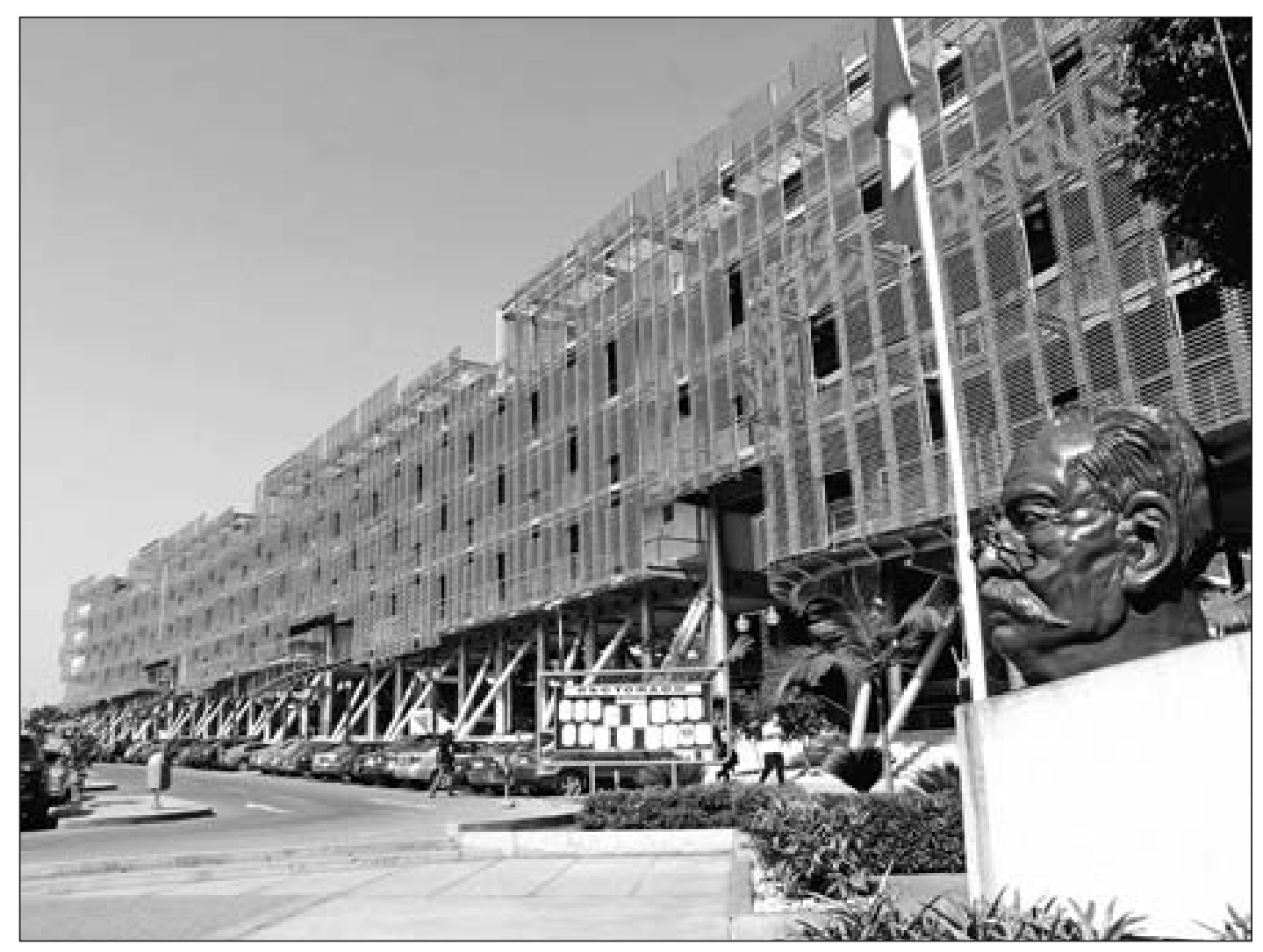


mutuamente. Por ejemplo, la vinculación con los grupos de interés ayuda a definir el perfil del egresado, lo cual ayuda muchísimo a la planificación curricular, a la investigación, a la proyección social y extensión universitaria, así como a la planificación estratégica.

3. El modelo de aseguramiento de la calidad propuesto está conformado por diversos sistemas; los más importantes son:

- Sistema de Gestión de la Calidad

- Sistema de Evaluación de la Enseñanza Aprendizaje

- Sistema de Evaluación de la Investigación

- Sistema de evaluación de la Extensión Universitaria

- Sistema de evaluación de Proyección Social

- Sistema de Cultura Organizacional

- Sistema de Información y Comunicación

- Sistema de Seguimiento al egresado

A los cuales se deben adicionar las políticas de calidad, los manuales de procedimientos y la auditoria de la calidad.

4. Una de las mayores ganancias de la acreditación ha sido la vinculación con los grupos de interés, pues nos ha permitido tener un contacto directo con la realidad de los mercados ocupacionales y el ámbito social del país, incrementando la pertinencia de nuestras actividades formativas, de investigaciones y proyección social y extensión universitaria.

\section{Referencias bibliográficas.}

Perú. Ley 28740 de Creación del Sistema Nacional de Evaluación y Acreditación y Certificación de la Calidad Educativa, publicado el 23 de mayo de 2006.

Perú. Reglamento de la Ley 28740 - SINEACE, DS 018 2007 -ED., publicado el 9 de julio de 2007.

Perú. Guía para Acreditación Carreras Profesionales Universitarias del CONEAU, del 16 de septiembre de 2009.

Perú. Modelo de Calidad para la Acreditación de Carreras Universitarias y Estándares para la Carrera de Educación. 2008.

Perú. Nueva Ley Universitaria. Ley 30220, Publicada el 09 de julio de 2014.

FACEE. Informe del Proceso de Acreditación de la Facultad de Ciencias Económicas y Empresariales 2012 -2014, Septiembre 2014.

Flores Konja, Alejandro y Manuel Hidalgo. La Norma ISO 9000 y el sistema de gestión de la calidad. En: Revista Quipucamayoq, de la Facultad de Ciencias Contables, Universidad Nacional Mayor de San Marcos. Vol. 22, No 41, Enero - Junio 2014.

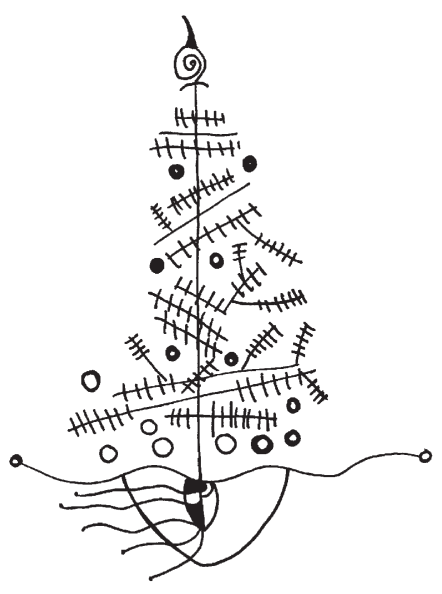

